\title{
A new concept in orthodontics: faster and healthier tooth movement by regularly consuming xyilitol chewing gum
}

\author{
Haryono Utomo \\ Department of Oral Biology \\ Faculty of Dentistry, Airlangga University \\ Surabaya - Indonesia
}

\begin{abstract}
Xylitol was first discovered in the 19th century, it wasn't until the 1960's that commercial production was first implemented. Recent studies showed that xylitol chewing gum is beneficial for preventing caries and periodontal disease. Therefore, it is also advantageous for orthodontic treatment, especially the fixed orthodontics patients who have difficulties in acquiring optimal oral health, particularly periodontal health which important in remodeling. However, how consuming xylitol chewing gum may stimulate tooth movement and preventing root resorption is still unclear. It is suggested that chewing activities may stimulate tooth movement, since jaw hypofunction leads to lower mineral apposition and bone function; and narrow periodontal ligament (PDL). These conditions may lead to impaired remodeling process, and increases the susceptibility of root resorption during orthodontic tooth movement. Moreover, since stimulation of the PDL could be mechanoreceptive (i.e. chewing action) or nociceptive (i.e. painful stimulation), periodontal nerve fibers are supposed to play an important role in bone remodeling. It is supported by a study which revealed that during tooth movement, the galanin-containing immunoreactive nerve fibers, a part of primary sensory neurons in the PDL is increasing. Galanin is able to induce osteoclast differentiation that needed for bone resorption in orthodontic treatment. The objective of this study is to elucidate a new concept in using xylitol chewing gum as an excellent media to have a faster and healthier orthodontic movement. Since continuous chewing stimulates the PDL which enhances tooth movement, improves oral health, and prevents root resorption; it is concluded that this concept is possible.
\end{abstract}

Key words: xylitol, chewing gum, orthodontic treatment

Correspondence: Haryono Utomo, c/o: Bagian Biologi Oral, Fakultas Kedokteran Gigi Universitas Airlangga. Jln. Mayjend. Prof. Dr. Moestopo no. 47 Surabaya 60132, Indonesia. E-mail: dhoetomo@indo.net.id, Telp. (031) 5053195

\section{INTRODUCTION}

Successful orthodontic treatment should include rapid tooth movement concomitantly with healthy periodontal tissues, and minimal root resorption. It is a common problem that plaque removal is difficult in individuals carrying orthodontic brackets and ligatures. In 1970, the dental importance of xylitol for dental plaque inhibition was discovered in Turku, Finland, followed by the launching of first commercial xyilitol chewing gums in $1975 .{ }^{1}$ After more than 30 years of xylitol dental use, unfortunately, lack of study had been investigated the advantageous effect of xylitol products to orthodontic treatment. There were studies which found that xylitol products were able to increase salivary $\mathrm{pH}^{2}$ and inhibit lactic acid formation ${ }^{3}$ in fixed orthodontic patients; therefore, reduces demineralization. However, the idea of consuming xylitol chewing gums to improve the quality of orthodontic treatment by promoting both periodontal health and rapid tooth movement; and also preventing root resorption is considered unusual.

The rate of tooth movement depends on the remodeling process. It could be affected by biological, mechanical or other factors i.e. force magnitude, age, sexual hormone, bone density, genetic variability, activation interval etc. ${ }^{5}$ There were some recent ideas to accelerate tooth movement rate such as "accelerated osteogenic orthodontics, AOO", also called Wilckodontics; nevertheless, it was complicated and still expensive (US\$ 10.000-15.000). ${ }^{6}$ Others were drugs that favor osteoclast recruitment and genetic engineering of cells involved in tooth movement, but it should take years and million of dollars of researches for commercial use in orthodontic treatment. ${ }^{5}$ Therefore, this new concept is regarded easier and more economical.

Owing to the remodeling process, a simple matter such as continuous chewing that may act as tooth movement stimulator was rarely discussed; thus it is considered as a new concept in orthodontic treatment. Some literatures supported the possibility of this concept. During tooth movement, it was found that the galanin-immunoreactive nerve fibers in periodontal ligament were increasing; galanin was able to stimulate osteoclast activity. ${ }^{7}$ In addition, the application of continuous force to the tooth also triggers addition bone volume, which enhances remodeling. ${ }^{8}$ Other theory is the hypofunctional periodontium concept which stated that in the absence or reduced occlusal function, the periodontium narrows, and its cushioning effect decreases which lead to increase vulnerability to root resorption during orthodontic movement. ${ }^{9}$ 
The objective of this review is to elucidate the possibility of using xylitol chewing gum as an appropriate media to stimulate tooth movement, optimizing dental and periodontal health, and simultaneously prevent root resorption during orthodontic treatment. Hopefully, this new concept could be included as a routine procedure during orthodontic treatment.

\section{Xylitol}

Xylitol is a 5-carbon sugar that was discovered by Fischer and Stahel in Germany, and Bertrand in France around 1890. Since the 1960s it was used for medical purposes such as parenteral nutrition and special dietary purposes. In 1970, the dental importance of xylitol for dental plaque inhibition was discovered in Turku, Finland, followed by the launching of first commercial xyilitol chewing gums in 1975, then USA a few months later. ${ }^{1}$

Until now, the use of xylitol chewing gums are widespread worldwide, that also supported by the launching of "Xylitol Oral Health Program" by the Academy of Dental Resources in 2006. ${ }^{10,11}$ However, researches of xylitol in orthodontics, especially in fixed orthodontics treatment were still unusual. One of the investigations was related to the effect of xylitol lozenges for increasing dental plaque $\mathrm{pH}^{2}$ and the other was the effect of xylitol tablets to streptococcus mutans and the formation of saliva lactic acid. ${ }^{3}$

Studies showed that 4 to 12 grams of xylitol per day are enough. The "all xylitol" mints and gums contain about one gram of xylitol in each piece. You could begin with as little as one piece four times a day for a total of four grams. It is not necessary to use more than 15 grams per day as higher intakes yield diminishing dental benefits. ${ }^{11,12}$

If used only occasionally or even as often as once a day, xylitol may not be effective, regardless of the amount. Use xylitol at least three, and preferably 5 times every day. Use immediately after eating and clearing the mouth by swishing water, if possible. Between meals, replace ordinary chewing gum, breath mints, or breath spray with comparable xylitol products. ${ }^{12}$

\section{Mastication and malocclusion}

Mastication is a complex task with numerous anatomical, physiological, and psychological components. Given the remarkable ability of individuals to adapt and compensate, a deficiency in one of the componenets can be compensated for by other components. For example, poor masticatory biomechanics might be compensated for by increased muscle strength. Masticatory ability, efficiency, and performance are three ways of measuring the capability of individuals to breakdown foods. Masticatory ability is a subjective measure or a perception, how well subjects think they break down foods. On the contrary, masticatory efficiency and performance are objective measures. ${ }^{13}$

Efficiency pertains to the number of masticatory cycles (i.e. number of chews) required to reduce foods to a certain size. ${ }^{13}$ Malocclusion negatively affects subjects' ability to process and break down foods. Compared to normal occlusion, the median particle sizes for Class I, Class II, and Class III malocclusions were approximately 9\%, 15\%, and $34 \%$ larger, respectively. Individuals with normal occlusion also produced a wider distribution of particles, which indicates better masticatory performance. In addition, malocclusion affects an individual's perception of how well they can chew. Compared with normal occlusion, individuals with Class III malocclusions reported the greatest difficulty, followed by Class II malocclusions and Class I malocclusions. ${ }^{14}$

\section{Biologic basis of tooth movement}

Orthodontic tooth movement is induced by mechanical stimuli and facilitated by remodeling of the periodontal ligament (PDL) and alveolar bone. A precondition for those remodeling activities, and ultimately tooth displacement, is the occurrence of an inflammatory process. Vascular and cellular changes were the first events to be recognized and described, and a number of inflammatory mediators, growth factors, and neuropeptides have been demonstrated in periodontal supporting tissues. Their increased levels during orthodontic tooth movement have led to the assumption that interactions between cells producing these substances, such as nerve, immune, and endocrine system cells, regulate the biological responses that occur following the application of orthodontic forces. ${ }^{15}$

Application of force to the tooth, could either increasing or decreasing the loading of the PDL. This strain is relayed to the osteoblasts in the bony socket lining. One of proteins in the membrane of the osteoblast is called an integrin. Integrins translate mechanical strain into a signal within the cell that can turn stimulate a gene to make the cell develop ligands. This "talk" between osteoblasts and osteoclasts is accomplished through a receptor activator of nuclear factor $\kappa$ B (RANK) and RANK ligand. RANK ligand on the osteoblast membrane which can interact with developing monocytes to cause them to differentiate into osteoclasts. Ligands allow intracellular communication, which stimulates undermining resorption, allowing tooth movement. Interestingly, other ligands can develop to block the RANK ligand and prevent osteoclast formation. ${ }^{4,5,15}$

However, this simple mechanism is modified by many other factors i.e. force magnitude, age, sexual hormone, bone density, genetic variability, activation interval etc. ${ }^{4,15}$ According literatures and practical experience, teeth move at different rates and there are differences between individuals. These differences can be experimentally demonstrated in dogs, where two to three times the amount of movement occurs in different dogs using the same force levels. The reason is beginning to be elucidated. ${ }^{5}$

The role of periodontal ligament nerve fibers in remodeling

The PDL is partly innervated by primary sensory neurons in the trigeminal ganglion. Trigeminal ganglion neurons are considered to transmit nociceptive and mechanoreceptive information from the periodontal ligament to the brainstem. 
Experimental tooth movement causes several changes in periodontal tissues such as the blood vessels, bone, and periodontal ligament. ${ }^{7,16}$

In the PDL, nerve fibers which contain calcitonin generelated peptide (CGRP) also increase in number during orthodontic tooth movement; numerous free nerve endings and perivascular endings appear in the apical region of the periodontal ligament. These nerve fibers are considered to be associated with the pain and discomfort that patients experience during orthodontic tooth movement. Therefore, it is possible that periodontal nerve fibers play a role in remodeling of the periodontal tissues during orthodontic tooth movement. ${ }^{7}$

Calcitonin gene-related peptide (CGRP) and galanin are known to have nociceptive functions in the spinal cord). CGRP can enhance nociceptive inputs to secondary neurons, while galanin has a suppressive effect on nociceptive transmission in the cord. Therefore, the increased galanin-immunoreactive neurons and coexpression of galanin and CGRP suggest that galanin is associated with the modulation of nociceptive information. CGRP has been suggested to affect bone remodeling during experimental tooth movement. This peptide is known to suppress differentiation of osteoclasts, and may promote bone formation. ${ }^{7,16}$

\section{Bone remodeling}

Bone remodeling is stimulated in a hierarchy. The chemical stimulation and interaction of osteoblasts and osteoclasts controls tooth movement. Factors such as prostaglandins like prostaglandin E2 (PGE2) and proinflammatory cytokines (i.e. tumor necrosis factor- $\alpha$, TNF- $\alpha$; interleukin- $1 \beta$, IL- $1 \beta$ ) can all be "up or down regulated" by hormones such as estrogen and are activated by environmental stimulation to remodel bone. Postmenopausal women tend to have a problem with increased bone resorption because estrogen levels are decreased in the body. ${ }^{5,15}$

\section{Factors that inhibit and increase tooth movement}

Chemical factors were identified that favor osteoclast recruitment so teeth can move faster. Some factors inhibit osteoclast activity and block inhibitors of osteoclast activation (i.e. RANK ligand inhibitor). Still other factors are known to promote osteoblastic activity and may help someday with increased anchorage for moving teeth (i.e. bone morphogenic protein, BMP). However, before these chemical applied to human, problems such as an appropriate delivery system need to be worked out and would involve questions of systemic vs. local delivery, dosage, effectiveness of the drug based on the delivery route chosen effect, side effects of the drugs etc. ${ }^{5}$

"Accelerated osteogenic orthodontics" also called Wilckodontics, has been done to move teeth faster. A slow speed bur is used to place dimples in the bone exposed by a flap procedure. This selective decortication is done and then the bone is covered with demineralized freeze dried bone and bovine Xenograft. This resulted in faster tooth movement and completion of an 18-month case in six months. ${ }^{6}$

\section{Root Resorption}

Root resorption is another unwanted effect or orthodontic treatment. Researches had been identified factors that predispose to root resorption. These are the following: a) root shape, longer and tapered roots are predisposed to more root resorption; b) amount of movement of the root apex; c) forces such as Class II elastics on upper canines; d) longer treatment times; e) Individual variation and genetic predisposition. ${ }^{5}$ Additionallly, periodontal hypofunction which manifestates as narrower periodontal ligament (PDL) has the propensity to increase root resorption by diminished cushioning effect. ${ }^{9}$

\section{DISCUSSION}

Studies by Sengun et al. and Steckesen-Blicks et al. supported the use of xylitol chewing gums to optimize dental and periodontal health during orthodontic treatment. ${ }^{2,3}$ Clinical studies have shown that chewing sugarless gum for 20 minutes following meals can help prevent tooth decay, i.e. by increase salivary $\mathrm{pH} .{ }^{17}$ In the future, look for chewing gum that delivers a variety of therapeutic agents that could provide additional benefits to those provided by the ability of gum to mechanically stimulate salivary flow. For instance, some gum might contain active agents that could enhance the gum's ability to remineralize teeth and reduce decay, or enable gum to help reduce plaque and gingivitis. ${ }^{18,19}$

Based on literatures, it had been found that chewing performance of young adults with excellent buccal segment occlusion is $40 \%$ better than that of "less than ideal" buccal segment relationship, and these differences increase with increasing number of chews. Moreover, masticatory performance also related to the classification of malocclusion, the greatest difficulty in chewing was Class III, followed by Class II and Class I respectively. ${ }^{14}$ Therefore, it is plausible that the majority of orthodontic patient already have masticatory hypofunction which has deleterious effects during orthodontic treatment such as the predisposition to root resorption, and poorer bone turnover or remodeling than normal occlusion. As the result, enhancing masticatory performance or occlusal function should be beneficial for a healthier root and periodontal tissues during tooth movement.

According to Deguchi et al. (2003) ${ }^{16}$ and Deguchi et al. (2006), ${ }^{7}$ CGRP in the periodontal nerve fibers has been suggested to affect bone remodeling during experimental tooth movement. This peptide is known to suppress differentiation of osteoclasts, and may promote bone formation; this action is counter-regulated by galanin. It is the peripherally transported galanin that plays a role in periodontal ligament remodeling. ${ }^{7,16}$ Consequently, 
continuous stimulation-inhibition impulses by consuming chewing gum, even though is not a nociceptive stimulus, could enhance PDL remodeling by up-regulate galanin synthesis in the trigeminal primary neurons innervating the periodontal ligament.

Referred to these studies, it is possible that the PDL stimulation may accelerate tooth movement. It is the chewing action that stimulates mechanoreceptor during gum chewing. However, the possibility of nociceptor stimulation could also be expected. During tooth movement there is an increase of pro-inflammatory mediators (i.e. prostaglandin E2, PGE2) which able to sensitize nociceptor and decrease pain threshold, and modulate pain perception during orthodontic treatment. ${ }^{15}$ Therefore, it is common that even light touch may elicit pain in moved teeth. Thus, occasionally, continuous chewing action such as gum chewing may act both as mechanoceptive and nociceptive stimulation which beneficial to stimulate orthodontic tooth movement.

Additionally, literatures have shown that the expression and production of some inflammatory mediators (PGE2, IL- $1 \beta$ are promoted by mechanical stimulation of the PDL. Cyclooxygenase2 (COX-2) is induced in PDL cells by cyclic mechanical stimulation and is responsible for the augmentation of PGE2 production. It was also demonstrated that compressive force up-regulated RANKL expression and induction of COX-2 in human PDL cells. ${ }^{15}$

Gum chewing is considered as cyclic mechanical stimulation. It is suggested that PDL cells under mechanical stimulation created by gum chewing may induce osteoclastogenesis through up-regulation of RANKL expression via PGE2 synthesis during orthodontic tooth movement, and resulted in faster tooth movement. It is in accordance with Garat et al., ${ }^{8}$ that light continuous bite force accelerates bone remodeling and prevents from excessive bone resorption. Therefore, it is logical that gum chewing is able both accelerates orthodontic tooth movement, and prevents from excessive bone resorption.

Despite the benefits of gum chewing, there is a possibility that it may cause TMJ problem. ${ }^{20}$ Even though it is still a controversy, precautions should be conducted. It must be considered that mastication or chewing activities related to many physiological factors, like size, composition and mechanical advantage of jaw-closing muscles, sensitivity of the teeth, muscle and TMJ can influence the generation of maximum bite force during mastication. It had been revealed that gender difference in bite force magnitude that boys being stronger than girls. Moreover, boys were able to withstand pain more than girls. These differences are particularly noteworthy for numerous forms of musculoskeletal pain, including fibromyalgia, temporomandibular dysfunction (TMD) and myofascial pain. ${ }^{21}$ High bite force magnitude in gum chewing is undesirable, especially for the TMD sufferers, since it could impair the TMD. Therefore, diagnosing TMD prior to continuous gum chewing activities during orthodontic treatment, especially in male patient, is mandatory.
Regarding to relationship between chewing activities and root resorption, a study related to hypofunctional periodontium by Sringkarnboriboon et al. ${ }^{9}$ also supported this concept. In this study, an experimental hypofunctional periodontal condition of hypofunctional teeth and control were applied with heavy and continuous orthodontic forces which naturally induce root resorption in teeth with normal periodontium. The result revealed that root resorption was greater in the hypofunctional periodontium group. This implies that factors other than the applied force were responsible for root resorption.

It was supposed that hypofunctional periodontium exhibited progressive atrophic changes in all functional structures, and accelerated the root destruction resulting from the mechanical stress of orthodontic force. Due to the narrow periodontal space in the hypofunctional periodontium, the applied force might concentrate in the compression area. In addition, the narrow periodontal space and derangement of functional fibers would eliminate the normal cushioning effect of the periodontal ligament, and resulting in a high concentration of force. ${ }^{9,22}$ As the result, continuous chewing may also prevent from root resorption and extensive bone resorption during orthodontic treatment

Concerning the variability (i.e. stickiness, consistency, taste, and price) of commercial xylitol chewing gum products, there should be researches investigating the most efficient characteristics for ideal orthodontic xylitol chewing gum. Stickiness is unfavorable characteristics for fixed orthodontic patients. In addition, durability of consistency is beneficial for maintaining adequate bite force to stimulate the PDL. However, it must be considered that gum chewing habit may lead to TMJ problem in certain individuals, especially who chews with one side only. ${ }^{20}$

The duration of chewing and hardness of chewing gums is also essential to be evaluated to prevent from pain, muscle fatigue and TMJ problem. ${ }^{22-24}$ In addition, besides gum chewing, it is plausible that other chewing actions could be done to stimulate the PDL. Several actions such as increasing chewing frequency during eating; intermittently occlude teeth or jaw clenching, which conducted with continuous light pressure between meals is considered able to give the same results as gum chewing.

For the concluding remarks, it should be noted that besides regularly consuming xylitol chewing gum; additional chewing frequency during eating food, and intermittent jaw clenching between meals are favorable for stimulating the PDL. Stimulated PDL resulted in an increase of periodontal health and a wider PDL, thus enhance the cushioning effect that able to reduce bone and root resorptions. Nevertheless, abusing gum chewing other than an adjunct of orthodontic treatment is prohibited. Moreover, instructing gum chewing to TMD sufferers is not recommended. Hence, it concluded that regularly consuming xylitol is beneficial for achieving safer and healthier orthodontic tooth movement. In order to refine this new concept, researches should be done to develop an effective orthodontic gum chewing method, 
to discover deleterious effect of continuous gum chewing which lasted for months or years, and inventing an ideal orthodontic chewing gum.

\section{REFERENCES}

1. DFI-xylitol. Xylitol history. Available online at URL http:// dficorp. net/xylitol/index.htm. Accessed November 2, 2007.

2. Sengun A, Sari Z, Ramoglu SI, Malkoc S. Evaluation of the dental plaque $\mathrm{pH}$ recovery effect of a xylitol lozenge on patients with fixed orthodontic appliances. Angle Orthod 2004; 74:240-4.

3. Steckesen-Blicks C, Lif Holgerson P, Olsson M, Bylund B, Sjo "stro“m I, Sko"ld-Larsson K, Kalfas S, Twetman S. Effect of xylitol on mutans streptococci and lactic acid formation in saliva and plaque from adolescents and young adults with fixed orthodontic appliances. Eur J Oral Sci 2004; 112: 244-8.

4. Graber TM, Vanardsdall RL, Vig KWL. Orthodontics: current principles and techniques. $4^{\text {th }}$ ed. St. Louis: Elsevier-Mosby; 2005. p. $266-80$.

5. Covell D. Can biology help us move teeth faster? Presented by Dr. David Covell, on Sept 20, 2004, Annual Session, Palm Springs. Available online at http:_www.pcsortho.org/bulletin/bulletin05/ Spring05pdfs/p\%2039-40\%20Covell.pdf.

6. Lynn S. AOO - Wilckodontics 1 - Speed Orthodontic Surgery. Arched Wire. 26 Mar. 2006. Available online at URL http://www.archwired. com/AOO1.htm. Accessed Nov 12, 2007.

7. Deguchi T, Yabuuchi T, Ando R, Ichikawa H, Sugimoto T, TakanoYamamoto T. Increase of Galanin in trigeminal ganglion during tooth movement. J Dent Res 85(7): 658-663. 2006.

8. Garat JA, Gordillo ME, Ubios AM: Bone response to different strength orthodontic forces in animals with periodontitis. J Periodont Res. 2005; 40: 441-5.

9. Sringkarnboriboon S, Matsumoto Y, Soma K. Root resorption related to hypofunctional periodontium in experimental tooth movement. J Dent Res. 2003; 82(6):486-90.

10. Lam M, Riedy CA, Coldwell SE, Milgrom P, Craig R: Children's acceptance of xylitol-based foods. Commun Dent Oral Epidemiol. 2000; 28: 97-101.
11. Ship JA, McCutcheon JA, Spivakovsky S, Kerr AR. Safety and effectiveness of topical dry mouth products containing olive oil, betaine, and xylitol in reducing xerostomia for polypharmacy-induced dry mouth .J Oral Rehab. 2007; 34: 724-32.

12. Xclearinc. How to use xylitol.. Available online at URL http:// www. xlear .com/xylitol/. Accessed Nov 19, 2007.

13. Buschang PH. Masticatory ability and performance: the effects of mutilatede and maloccluded dentitions. Seminars in Orthodontics 2006; 12(2): 92-5.

14. English JD, Buschang PH; Throckmorton G. Does malocclusion affect masticatory performance? Angle Orthod 2002; 72(1): 21-7.

15. Yamaguchi M, Kasai K. Inflammation in periodontal tissues in response to mechanical forces Arch Immunol Ther Exp, 2005; 53:388-98.

16. Deguchi T, Takeshita N, Balam TA, Fujiyoshi Y, Takano-Yamamoto $\mathrm{T}$. Galanin-immunoreactive nerve fibers in the periodontal ligament during experimental tooth movement. J Dent Res. 2003; 82(9): 677-81.

17. Polland KE, Higgins F, Orchardson R. Salivary flow rate and $\mathrm{pH}$ during prolonged gum chewing in humans. J Oral Rehab 2003; 30; 861-65.

18. Dawes $\mathrm{C}$, Kubieniec $\mathrm{K}$ The effects of prolonged gum chewing on salivary flow rate and composition. Arch Oral Biol. 2004; 49(8): 665-9.

19. American Dental Associaton. Chewing gum. Available online at URL http://www.ada.org/ada/seal/index.asp. Accessed Nov 20, 2007.

20. Miyake R, Ohkubo R, Takehara J, Morita M. Oral parafunctions and association with symptoms of temporomandibular disorders in Japanese university students. J Oral Rehab 2004; 31:518-23.

21. Berkley KJ. Zalcman SS. Simon VR. Sex and gender differences in pain and inflammation: a rapidly maturing field Am J Physiol Regul Integr Comp Physiol. 2006; 291:R241-4.

22. Verna C, Dalstra M, Melsen B. Bone turnover rate in rats does not influence root resorption induced by orthodontic treatment. Eur J Orthod. 2003; 25(3):359-63.

23. Bonjardim LR, Gavia MBD, Pereira OLJ, Castelo PM. Bite force determination in adolescents with and without temporomandibular dysfunction. J Oral Rehab. 2005; 32:577-83.

24. Farella M, Bakke M, Michelotti A, Martina R. Effects of prolonged gum chewing on pain and fatigue in human jaw muscles. Eur J Oral Sci 2001; 109:81-5. 